\title{
CT and PET Image Registration: Application to Thorax Area
}

\author{
Davood Khodadad \\ Experimental Mechanics, Luleå University of Technology SE-971 87, Luleå, Sweden \\ Email: davood.khodadad@ltu.se
}

Alireza Ahmadian

Institute for Advanced Medical Technologies (IAMT), Tehran University of Medical Science, Tehran, Iran

Email: ahmadian@sina.tums.ac.ir

Hossein Yousefi Banaem

Department of Medical Physics and Medical Engineering, Isfahan University of Medical Sciences, Isfahan, Iran

Email: hossein_yousefy@yahoo.com

\author{
Mohammad Reza Ay and Armaghan Fard-Esfahani \\ Research Institute for Nuclear Medicine, Tehran University of Medical Science, Tehran, Iran \\ Email: $\{$ mohammadreza_ay, fardesfa $\} @$ tums.ac.ir
}

\begin{abstract}
Accurate attenuation correction of emission data is mandatory for quantitative analysis of PET images. One of the main concerns in CT-based attenuation correction (CTAC) of PET data in multimodality PET/CT imaging is misalignment occurred due to respiratory artifact between PET and CT images. In this paper a combined method which is simple and fast is proposed for registration of PET and CT data to correct the effect of this artifact. The algorithm is composed of two step: First step is meant to reduce the noise by applying an adaptive gradient anistropic diffusion filter then using Iterative closest point (ICP) registration method in order to obtain initial estimation to ensure fast and accurate convergence of the algorithm. At the second step, the respiratory related artifact of PET images is greatly reduced by employing Free Form Deformation algorithm based on $B$-spline which provides more accurate adaptive transformation to align the images.
\end{abstract}

Index Terms-registration, PET, CT, thorax, FFD, CTAC, gradient anistropic diffusion filter

\section{INTRODUCTION}

Image registration is overlaying at least two set of images, reference image and float image in order to obtain a transformation between the images specially correspondence and coordinates from a reference image to coordinates of homologous point in a test image[1]. In image analysis and diagnostic clinical application field, in order to compare or integrate the information which is gained from various data sources like in multimodality systems, the image from different time or different field of views and multichannel image restoration, registration is an important parts of process [2]-[4].

Manuscript received November 6, 2013; revised February 10, 2014
The computed tomography (CT) scan of the thorax has been widely used for investigating and staging of lung tumors. It has the advantage of offering high resolution images with detailed anatomical structures, but its main drawback is lack of pathophysiologic information of the tumors. In contrast to CT, positron emission tomography (PET) produces images reflecting physiologic of tissues. However for the photon attenuation, the spatial resolution of detectors and breathing related motions, these images have problem in achieving accurate lesion size and shape as they are typically noisy and blurry [5], [6].

Registration of PET and CT could lead to accurate differentiation of viable tumors from being masses, radiotherapy planning and monitoring treatment response and cancer staging [7].

Although a combined PET/CT automatically removes many of misalignments but breathing related non-rigid mismatches still persist during hybrid imaging. However, despite of advantages of CTAC in improving the accuracy of quantification in PET images, this method sometimes introduces an additional risk for artifacts on PET images specially when there is some level of misalignment between emission (PET) and transmission (CT) data.

Although there is a claim that the misalignment in PET/CT systems is minimum due to its hardware fusion concept but it should be emphasized that there is respiratory motion artifact in up to $96 \%$ of combined PET/CT examinations [2], [7]. In order to minimize the mismatch between CT and PET, developing of softwarebased image registration seems to be mandatory to increase the accuracy of CTAC.

In thorax and abdominal regions, the respiratory related motions are a major obstacle to handle and 
achieve satisfactory levels of registration reproducibility and accuracy [8].

Linear registration is not sufficient to cope with local deformation produced by respiration [9]. Non-linear and non-rigid registration methods remain necessary to compensate and avoid for the vast cardiac and respiratory motions. Free Form Deformations (FFD), originally introduced by [10] and Non-rigid FFD technique has been used as an effective method to perform image registration [11] in breast MR images. To motivate our choice of B-splines [12] as the most adequate basis functions among polynomials, wavelets and radial basis functions, to represent the deformation, some points should be considered. First it has small overlap, as a result makes faster algorithm and reduce the interdependency between the parameters.

Second, B-splines have the least number of contributing functions respect to polynomials, radial basis functions, and wavelets that makes it faster. B-spline on the other hand using FFD with support of local control provides us more accurate adaptive transformation to align the images. Non-rigid B-spline FFD was chosen because of the flexibility of FFD which comes from the fact that no assumptions on the underlying anatomy are made and its fastness in comparison with other elastic registration models such as fluid models.

The various motions and the variability of the organs make unpractical to choose a more constrained model of the organs in thoracic oncology application. But special constraints must be added to convergence toward a proper registration [13], [14]. In our proposed method an adaptive low pass filter followed by segmentation step are applied to identify corresponding structures in both modalities. This approach provides us with the necessary initial constraint to guide the algorithm and prevent from getting trapped in local minima of the chosen similarity criterion.

\section{MethodS AND MATERIAL}

Our data sets for this paper consisted of CT and PET images of ten patients. CT images have a size of $512 \mathrm{x}$ $512 \times 138$ with resolution of $1 \times 1 \times 5 \mathrm{~mm}^{3}$ and PET images of a size $168 \times 168 \times 320$ with resolution of $4 \times 4$ x $3 \mathrm{~mm}^{3}$. See Fig. 1. As Fig. 2 shows, the general scheme of proposed algorithm consists of the following stages.

\section{A. Pre-Processing Stage}

Before image registration, the CT images were resampled and the volume extents and pixel sizes were adjusted to equalize PET images. The choice of proper type of resampling technique between PET and CT depends on the trade-off between the desired accuracy of the interpolation and computational complexity. To improve the segmentation results in PET images, the noise should be reduced. The issue of denoising in PET images is a challenging task due to the inherent low quality and low signal to noise ratio of PET data. Most of the standard denoising methods will fail when they encounter with high noise level which cannot removed by applying classical averaging filters. Also averaging methods destroy anatomical information and structures as well as boundaries and edges.
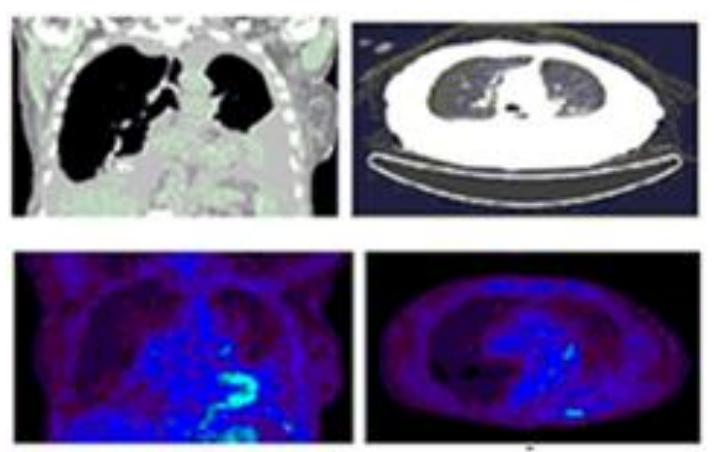

Figure 1. Coronal and axial slice of original data volume (CT)-first row left-first row right. Coronal and axial slice of PET-second row-left and right).

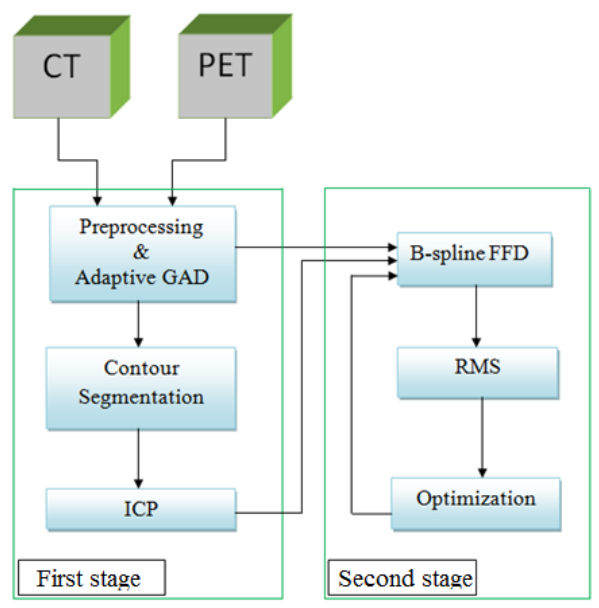

Figure 2. Schematic of proposed method

As the accuracy of our proposed algorithm relies on preserving the anatomical features and edges, a proper filter with good performance in keeping the image details should be chosen. Among the existing choices Brushlet denoising has shown an acceptable ability of removing background noise while enhancing the functional data over wavelet denoising.

Gradient Anistropic Diffusion image filter (GAD) is an efficient smoothing algorithm by introducing conductance parameter as a measure of image smoothness which is related to the gradient magnitude. This filter changes GAD conductance parameter adaptively to achieve more diffusion in the interior regions, where the gradient is small and less diffusion at the edges where the gradient becomes larger. So it has the ability of reducing the noise while keeping the anatomical information and improving the functional information.

This filter requires 3 parameters:

$$
\frac{\partial I(x, y, t)}{\partial t}=\operatorname{div}[(g\|\nabla I(x, y, t)\|) \nabla I(x, y, t)]
$$

Using the original image $\mathrm{I}(\mathrm{x}, \mathrm{y}, 0): \mathrm{R} 2 \rightarrow \mathrm{R}+$ as the initial condition, where $\mathrm{t}$ is an artificial time parameter 
$\|\nabla I(x, y, t)\|$ is the gradient magnitude and $\mathrm{g}\|\nabla I(x, y, t)\|$ is an "edge stopping" function.

The noise statistics is strongly spatially-variant and have shown a Poisson behavior in PET images [15]. But as we need to preserve physiological and functional information of PET image [14], therefore different regions of PET need to be smoothed differently. To perform this the proposed filter estimates the diffusion in background and change it through background by local estimation of the noise obtained by applying local median filter. By this adaptive behavior of GAD, the amount of smoothing and noise reduction will be locally controlled even in the interior region, therefore preserving the functional and physiological information.

\section{B. Image Segmentation}

To segment the thoracic area in the PET images, an adaptive thresholding algorithm based on the minimum and maximum intensity of each image is applied [16], [17]. By applying this step, unwanted boundaries and structures are removed from the image, thus provides much faster algorithm avoiding redundant calculations.

\section{Initial Registration}

After segmentation of structures (lungs) their contours are extracted (Fig. 3) and superimposed to Iterative Closest Point, ICP registration algorithm. See schematic of method in Fig. 2. The ICP algorithm iteratively tunes a rigid transformation to minimize Euclidian distance between two element (point, line, and triangle) sets. The advantage of using ICP is due to the fact that it always converges in a monotonous and fast way towards the closest registration position.

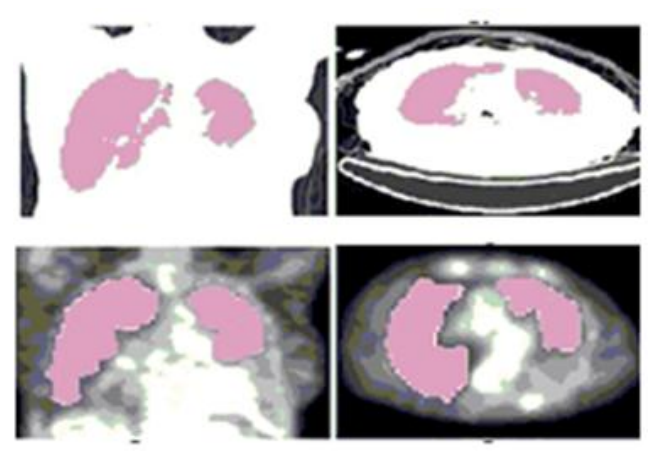

Figure 3. First row: segmented CT, coronal (left) and axial (right). Second row: segmented coronal PET (left) and segmented axial PET (right).

Once the ICP step is done, one can easily define the candidate points or areas to become suitable landmarks. The output features of the initial registration step were applied to the PET and CT image. This information plays an important role as constraints and conditions that should be met in the FFD registration step. This initialization will help in smaller searching area in the final solution thus, preventing from getting trapped in local minima of the chosen similarity criterion. This step (first stage in Fig. 2) gives us the initial constraints that will be used in FFD algorithm in second stage to import initial convergence and to increase the overall speed of the algorithm by reducing the complexity and defining initial condition of transformation. Without it, we will encounter misregistration due to the lack of proper initial conditions.

\section{B-Spline FFD Registration}

To the registration purposes, FFD is computed by means of linear combination of splines basis function as a particular case of non-linear transformation. In this technique deformation of the test image is achieved by tuning an underlying mesh of control points. As justified in section 1, B-spline based free form deformation has been chosen to cope with all possible deformations that may encounter in the mentioned application. Once the FFD grid has been optimized, control point (CP) displacements are interpolated to obtain a smooth and continuous $\mathrm{C}^{2}$ transformation [12].

Due to the compact support and separablity properties of B-spline they can be pre-calculated and stored in an array to accelerate the process. Moreover Bsplines are scalable in the sense that any coarse level deformation can be represented at a finer scale without any loss of information [12].

\section{E. Optimization}

When the transformation model is chosen, the similarity criterion that will drive the optimization of $\mathrm{CP}$ displacements must be defined. As the labeled images with linear intensity relation are used, the root mean square (RMS) difference of the corresponding pixel intensity, summed across the whole image, are used in order to determine the optimal deformation parameters. Optimization of the transformation parameters (for example control point displacement), is achieved by applying iteratively a gradient estimation to all control points simultaneously as proposed by [12] along the gradient direction until no further improvement of the similarity measure is found. At each iteration, finite difference of local gradient estimation is computed for each control points. Furthermore a local spring force regularization term has been added to pull each nodes towards the centriod of its neighboring nodes to keep with the nodes from intersecting, which could lead to unwanted alteration of structure topology[18], [19].

It should be point out that employing RMS as similarity criterion allows to deal with several structures in each image at the same time to perform the registration.

\section{RESULT}

The desired accuracy of registration depends on several factors such as image modalities, spatial resolution or anatomical regions and nature of its deformation involved in the process. In this work, the required accuracy should be reasonably high, due to the lack of identifiable structures in thoracic region and its severe deformation. The chosen accuracy must also assure a correct classification with respect to visualized tumors. Desired accuracy must have been chosen commensurate with the poorest spatial resolution, which is PET image resolution in this paper.

In our application, visual inspection (Fig. 4) by overlay chessboard image allows our expert to investigate 
the registration result for most important anatomical structures and control points.

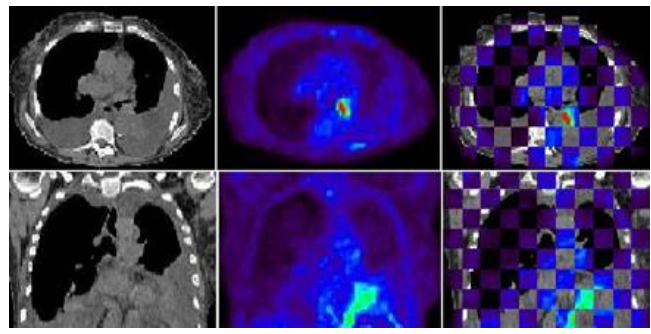

Figure 4. Example of 2D axial (top) and coronal (bottom) slice of the CT (left), registered PET (center) volumes and the chessboard display (left).

A quantitative analysis was also carried out to evaluate the accuracy of the ICP, free form deformation and its constraint imposed form initial registration information. To this, we have computed an overlap measure OM, between the reference and registered structure (lung). Overlap measure as a classical criterion, becomes unity if total overlap or perfect registration is achieved.

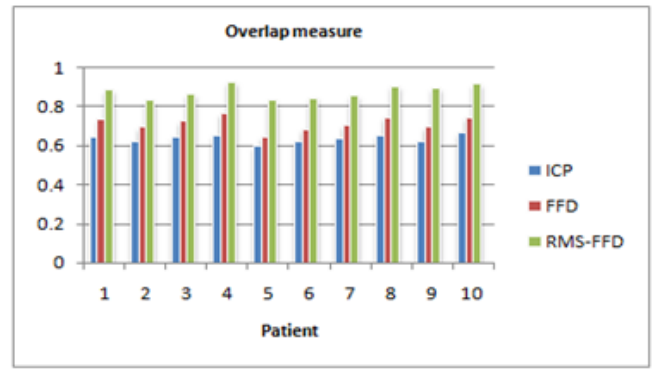

Figure 5. Summary of the results for OM calculation from applying the algorithm on 10 data sets

Fig. 5 summarizes the results of calculating OM obtained from applying the algorithm on 10 data sets. As it is seen, ICP method has failed to produce acceptable results due to lack of its ability to handle the local deformations. While applying FFD has improved the performance of registration significantly. Average of $\mathrm{OM}$ value obtained by ICP and FFD are 0.63 and 0.71 , respectively. This value is raised to 0.87 by means of applying our proposed two stage method using RMS-FFD algorithm This clear improvement of the results by an order of $16 \%$ has been achieved when the initial constraints superimposed to the FFD which is due to the power of constrained FFD to converge to the best solution and confirmed by our expert using visual inspection.

In addition to registration accuracy, computational cost of the method was also analyzed. As expected the inclusion of the initial constraints allows us to speed up the overall process. The average computing time by simple FFD was found around $470 \mathrm{~ms} / \mathrm{pix}$. When constraining the FFD by means of two step algorithm using initial registration information, this computational time considerably reduces by order of $34 \%$.

Also the CP distances from each other (grid size) were varied uniformly to investigate its effect on the accuracy of registration and the performance time. The results in Fig. 6 shows that although by decreasing the $\mathrm{CP}$ distance (equal to increasing number of $\mathrm{CP}$ and grid size) the accuracy of registration is improved but around the distance of $20 \mathrm{~mm}$ the computational rate increases quickly (39\%) while the accuracy does not show any significant increase. This evaluation shows the best choice for $\mathrm{CP}$ distance from each other experimentally is found as $20 \mathrm{~mm}$ and $15 \mathrm{~mm}$ (for CTAC purposes). Decreasing distance lower than $20 \mathrm{~mm}$ does not significant effect on accuracy while computational time increases.
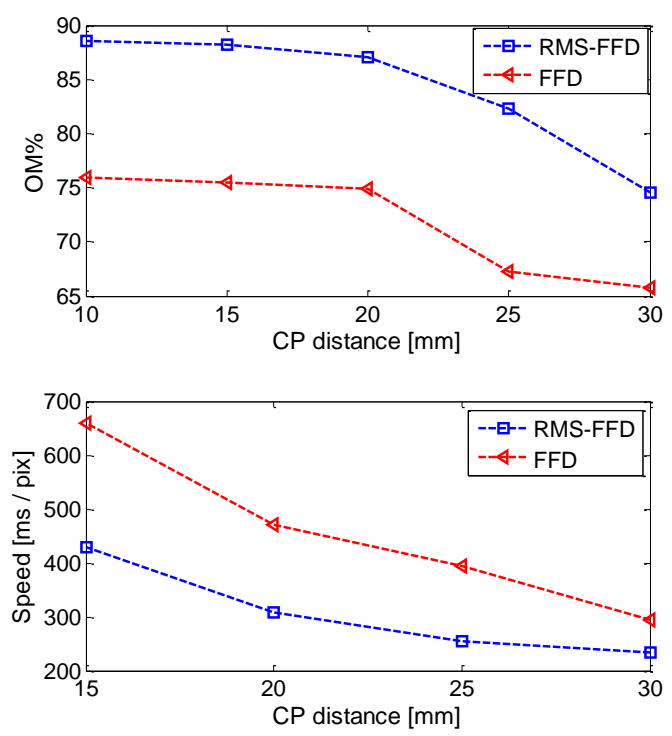

Figure 6. Relation between accuracy and CP distance from each other. Decreasing distance lower than $20 \mathrm{~mm}$ (by increasing grid size of CP's) does not significant effect on accuracy while computational time increases severely.

\section{COCLUSION}

Image registration is an important task that provides the ability of visual and diagnosis analysis of images acquired at different occasions by different modalities. In this paper a fast and accurate registration algorithm is presented that can cope with many deformation and severe breathing related motions in thoracic region based on B-spline free form deformation. The new idea of applying adaptive GAD filter before segmentation and using anatomical information directly obtained from the image at the first stage to take the necessary constraints for FFD at the second stage has significantly improved the registration performance by $34 \%$ as compared with simple FFD by which the accuracy of registration is almost $16 \%$.

As the results reasonably proved that our proposed method can be chosen as an efficient algorithm for data analysis in thoracic region and other applications with sever deformation and motions artifacts such as avoiding erroneous attenuation correction for PET reconstruction. The non-rigidity in the image and various local deformation is effectively modeled by means of B-spline based free form deformation which is clearly confirmed by the significant difference in value of overlap measure between ICP and FFD.

The proposed method can be further used for reducing the level of mis-registration between CT and PET images before applying attenuation correction in order to reduce 
the CTAC artifacts arising from data mismatch. Also it helps to reduce the radiation dose in PET/CT by avoiding repetition of CT imaging. These advances increase the potential of the method for routine clinical application. The method still needs more validation and assessment in clinical environment and extensive phantom study which is currently undergoing.

\section{REFERENCES}

[1] L. G. Brown, "A survey of image registration techniques," ACM Comput. Surv., vol. 24, pp. 325-376, 1992.

[2] W. V. Vogel, J. A. van Dalen, B. Wiering, H. Huisman, F. H. Corstens, T. J. Ruers, et al., "Evaluation of image registration in $\mathrm{PET} / \mathrm{CT}$ of the liver and recommendations for optimized imaging," Journal of Nuclear Medicine, vol. 48, pp. 910-919, 2007.

[3] M. Marinelli, V. Positano, F. Tucci, D. Neglia, and L. Landini, "Automatic PET-CT image registration method based on mutual information and genetic algorithms," The Scientific World Journal, vol. 2012, 2012

[4] M. Marinelli, V. Positano, S. G. Nekolla, P. Marcheschi, G. Todiere, N. Esposito, et al., "Hybrid image visualization tool for 3D integration of CT coronary anatomy and quantitative myocardial perfusion PET," International Journal of Computer Assisted Radiology and Surgery, pp. 1-12, 2013.

[5] W. P. Segars, B. M. Tsui, A. Da Silva, and L. Shao, "CT-PET image fusion using the 4D NCAT phantom with the purpose of attenuation correction," in Nuclear Science Symposium Conference Record, 2002 IEEE, 2002, pp. 1775-1779.

[6] H. Lee, J. Lee, N. Kim, I. K. Lyoo, and Y. G. Shin, "Robust and fast shell registration in PET and MR/CT brain images," Computers in Biology and Medicine, vol. 39, pp. 961-977, 2009.

[7] R. Shekhar, V. Walimbe, S. Raja, V. Zagrodsky, M. Kanvinde, G. $\mathrm{Wu}$, et al., "Automated 3-dimensional elastic registration of whole-body PET and CT from separate or combined scanners," Journal of Nuclear Medicine, vol. 46, pp. 1488-1496, 2005.

[8] X. Liu, "Shape-correlated statistical modeling and analysis for respiratory motion estimation," Ph.D. dissertation, University of North Carolina, 2011.

[9] H. Yousefi, A. Ahmadian, D. Khodadad, H. Saberi, and A. Daneshmehr, "An optimised linear mechanical model for estimating brain shift caused by meningioma tumours," International Journal of Biomedical Science and Engineering, vol. 1, pp. 1-9, June 10, 2013.

[10] T. W. Sederberg and S. R. Parry, "Free-form deformation of solid geometric models," in ACM Siggraph Computer Graphics, 1986, pp. 151-160.

[11] D. Rueckert, L. I. Sonoda, C. Hayes, D. L. G. Hill, M. O. Leach, and D. J. Hawkes, "Nonrigid registration using free-form deformations: Application to breast MR images," IEEE Transactions on Medical Imaging, vol. 18, pp. 712-721, 1999.

[12] J. Kybic and M. Unser, "Fast parametric elastic image registration," IEEE Transactions on Image Processing, vol. 12, pp. $1427-1442,2003$.

[13] O. Camara, G. Delso, and I. Bloch, "Evaluation of a thoracic elastic registration method using anatomical constraints in oncology," in Proc. 24th Annual Conference and the Annual Fall Meeting of the Biomedical Engineering Society EMBS/BMES Conference on Engineering in Medicine and Biology, 2002, pp. 1011-1012.

[14] D. Khodadad, A. Ahmadian, M. Ay, A. F. Esfahani, H. Y. Banaem, and H. Zaidi, "B-spline based Free Form Deformation Thoracic non-rigid registration of CT and PET images," in Proc. of SPIE Vol, 2011, pp. 82851K-1.

[15] J. Geng, Y. Chen, D. Yin, J. Tian, and S. Chen, "Noise components on positron emission tomography images," BioMedical Materials and Engineering, vol. 13, pp. 181-186, 2003.

[16] H. Y. Banaem, A. Ahmadian, H. Saberi, A. Daneshmehr, and D. Khodadad, "Brain tumor modeling: Glioma growth and interaction with chemotherapy," in Proc. SPIE Vol, 2011, pp. 82851M$82851 \mathrm{M}-6$.

[17] H. Yousefi-Banaem, S. Kermani, O. Sarrafzadeh, and D. Khodadad, "An improved spatial FCM algorithm for cardiac image segmentation," in Proc. 13th Iranian Conference on Fuzzy Systems, 2013, pp. 1-4.

[18] D. M. J. Vorsatz, Dynamic Remeshing and Applications, 2006.

[19] J. Vorsatz, C. Rössl, and H. P. Seidel, "Dynamic remeshing and applications," in Proc. 8th ACM Symposium on Solid Modeling and Applications, 2003, pp. 167-175.

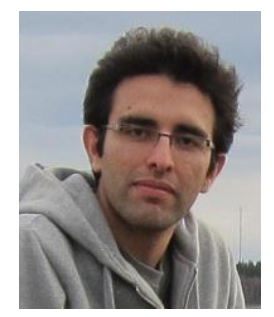

Davood Khodadad received his BS and MS degrees in Bioelectrical Engineering from Sahand University of Technology, Tabriz, and Tehran University of Medical Sciences, Tehran, in 2008 and 2011, respectively. He is currently active as a PhD student in the Division of Experimental Mechanics at Luleå University of Technology, Sweden. His research interests include optical metrology, imaging and image formation, and signal and image processing. His research is currently focused on development of pulsed multispectral digital holography for three-dimensional imaging.

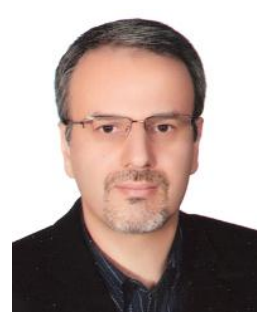

Alireza Ahmadian received his $\mathrm{PhD}$ in Biomedical Image Processing from the Imperial College of Science and Technology in Medicine, London, in 1997. He was at the King's College London as a research associate during 1999-2001. He is currently holding the chair of Department of Biomedical Systems \& Biophysics at the Tehran University of Medical Sciences. He is also Head of Image Guided Surgery Systems at the Institute for Advanced Medical Technologies (IAMT) since 2009. He has authored or coauthored over 120 papers in international journals and conferences.

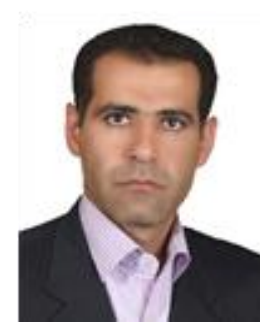

Hossein Yousefi-Banaem was born in Urmieh, Iran, in 1979. He received the BS degree in Radiology of technology from the Tabriz University of Medical Science, Tabriz Iran 2006, and the MS degree in medical engineering from Tehran University of Medical Science (TUMS) Tehran, Iran in 2011. Currently he is a PhD. Student of medical engineering at Isfahan University of Medical Science (MUI) Isfahan, Iran. His current research interests include Medical Image Processing and Analysis, Tissue Modeling and Cardiac MRI.

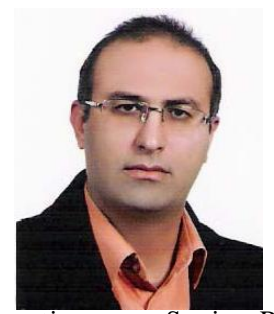

Mohammad Reza Ay received his PhD in Medical Radiation from Tehran Polytechnic University, Tehran, Iran in 2004. He also received his $\mathrm{PhD}$ in Medical Physics Geneva University, Geneva, Switzerland in 2006. Dr. Ay is currently Head of Research Center for Molecular and Cellular Imaging, Institute for Advanced Medical Technologies, Tehran University of Medical Sciences. He is also active as a Senior Researcher in the PET Instrumentation and Neuroscience Lab, Geneva University Hospital, Switzerland. He received the recipient of a 2013 first rank on Innovators and Inventors in 18th Razi International Festival in Medical Sciences and 2006 IEEE MIC Trainee Award. He has authored or coauthored over 120 papers in international journals and conferences and contributed to three books.

Armaghan Fard-Esfahani, is professor of school of nuclear medicine, Tehran University of Medical Sciences since 2011. She received M.D. degree from Tehran University of Medical Sciences in 1990. She was research fellow of Positron Emission Tomography, University of Toronto, Sunnybrook Hospital, Canada, during 2007 and 2008. She has authored or coauthored more than 150 papers in international journals and conferences. Her research interests include nuclear medicine, Positron Emission Tomography, Cardiac SPECT, Gated Cardiac SPECT and Computer Applications in Nuclear Cardiology. 